\title{
Change Process, Supports and Barriers in Residential Treatment Program: A Qualitative Approach
}

\author{
Katerina Flora \\ Panteion University of Social and Political Studies, Athens, Greece \\ Email: katerinaflora@hotmail.com
}

Received 29 August 2014; revised 4 October 2014; accepted 6 November 2014

Copyright (C) 2014 by author and OALib.

This work is licensed under the Creative Commons Attribution International License (CC BY). http://creativecommons.org/licenses/by/4.0/

(c) (i) Open Access

\begin{abstract}
Various studies have employed quantitative and qualitative methods in order to explore the process of behavioral change emerging during a drug addiction treatment, i.e. the factors both encouraging and discouraging such change. Treatment has been conceived as a process of maturation ("maturing out" theory) or as a sudden behavioral change ("rock bottom experience"). This article focuses on the qualitative approach of the therapeutic procedures of a residential treatment program. The participants of the study were 46 clients undergoing a substances treatment. In order to collect the data, we used the semi-structured interview. All conversation data were taped and later on elaborated with the help of the contextual content analysis. Three main categories derived from these analyses: the supports and the barriers clients face, as well as the way they handle the idea of changing during their addiction treatment. The findings highlight the importance of communality within such a framework, emotional management, practical matters the addicts must face, as well as the contribution of personal characteristics, such as strong will and determination. The implications of the study for clinical practice and future research are still debated.
\end{abstract}

\section{Keywords}

Treatment, Supports, Barriers, Change

Subject Areas: Psychology, Sociology

\section{Introduction}

The recovery process from substance abuse has been the object of numerous studies in the past 50 years, since Winick (1962) [1] first presented his "maturing out" theory. Following this theory, substance abusers mature 
while adopting grown-up roles and responsibilities at about their mid forties. Although Winick's thesis has been widely analyzed by researchers, it rather explores only certain parts of the truth about recovering. In the second most popular theory, belonging to Waldorf (1983) [2], recovery is being described as the result of a "sudden change" in a person, mainly attributed to a series of negative experiences, e.g. imprisonment, homelessness, ruptured family bonds, which can be of great importance to the individual so as to render recovery the only possible way out. This sudden behavioral change is called by others, like Maddux \& Desmond (1980), the "rock bottom experience", while others see it as an existential realization or a "flicker" [3].

Actually, there may be no antithesis between the beliefs that a person's behavior changes after an unexpected apperception and that people can change in the course of time and while they mature. Vaillant $(1966,1983)[4]$ [5] notices that not only former heroin abusers but former alcoholics as well came to a particular point in their lives, in which they decided to quit drugs or alcohol respectively. Until that special moment comes, Vaillant remains doubtful over the decisive role personal experiences can play and prefers instead to observe the maturation of powers that had been incubating for months or even years. Whatever means they might use in order to quit their addiction, clients very often find themselves reconstructing their lives in order to lead a sober way of life. Some experts believe that behavioral changes are deliberately adjusted to an abuser's decision of moving on with his/her life, while others claim that they are already in progress before a person starts recovering from his/her addiction [6].

According to Prochaska, DiClemente and Norcross (1992) [7], clients asking for help wonder whether a change in their lives is necessary or if it is really possible for them to change. Their own answers show that substance abusers must first tackle such issues before aiming at reaping the effectiveness of behavioral change [7]. The "action orientated" approach most treatment programs present covers the needs of those clients, who are already well prepared to attempt some changes in their lives. In this way, these programs show poor results with most clients, especially those undergoing an addiction treatment for the first time and are still at odds with the possibilities of change [8].

Older research projects conducted on substance abusers, who have managed to change by themselves or by undergoing treatment, revealed a series of factors molding therapy and change during treatment [2] [9]. The factors emerging in these studies are characteristic of therapy and represent the basic components of human functionality: improvement of close interpersonal relations, behavioral change within social networks, increased job offers and other work issues, interest in health condition, as well as more catholic factors, such as maturity and the sense of whom the client is and would want to be.

These factors symbolize the rewards that replace the addiction problem or are lost on those clients who fail to master their addictive behavior. In addition, subjective factors, such as personal responsibility, identity, personal values and satisfaction gained from a certain activity, are also deemed as highly important in treating addiction [10].

Recent findings have shown that behavioral changes, after participating in a residential treatment program, are characterized by an increase in experiencing positive feelings, the sense of personal strength and freedom [11]. Furthermore, the same study shows that clients gained more self-esteem, whereas changes were less attributed to fatalistic causes. This can be interpreted as a successful transition to a higher level of autonomy, a broader outlook on life and a boost of realism.

On the other hand however, studies carried out on self-help groups using the "twelve steps" method (e.g. FelixOrtiz, Salazar, Gonzalez, Sorensen, Plock, 2000) [12] came to the conclusion that supporting and understanding the group while giving it freedom of speech can vastly contribute in the recovery process, mood improvement and decrease of substances abuse.

Summing up the above mentioned theses, one could say that the change during the addiction treatment is mostly connected to the development of positive characteristics in the behavior of the individual, whereas the social network plays indisputably a decisive role in choosing and maintaining a course of action [13]. The recovery process from substances within a structured therapeutic framework, such as in the case of a residential treatment program, includes not only general descriptions of the recovery experience but details about the treatment itself (concept, structure and content).

The term "supports" describes all those factors aiming at a positive therapeutic outcome (in the case of a residential treatment program this concerns the staying in, continuing and completing of treatment). Depending now on the form of treatment employed, these factors are subject to change. However, it has been noted that there are some common characteristics found in the very nature of addiction and that theses may concern all or most 
treatment forms. Factors promoting the positive therapeutic outcome of treatment include personal characteristics, psychological parameters, social circumstances or characteristics of the chosen form of treatment. The common characteristic of all those factors is that they enhance the client's motivation to change and render make staying in treatment seem easier. "Supports" act usually as counterweight of the many and different hardships an abuser must face within his effort of mastering his addiction and keep him in treatment. "Supports" include the positive therapeutic alliance between the client and the counselor, the empathic understanding of the client on behalf of the counselor, the commitment of the client to the therapy, the positive interpersonal relations, the client's need/ wish to change and the reasons behind this wanted change. Furthermore, the therapeutic effort can be enhanced by other cyclical factors and events which add to the motivation for change [14].

"Barriers", on the other hand, describe conditions or features of the individual or his/her social environment, which discourage the client while entering and during treatment. These obstacles can prevent a client from considering treatment in the first place or lead to poor therapeutic results, given that the client has already began a therapeutic program. The barriers most commonly employed by clinical researchers of the field include interaction, social support, practical difficulties (e.g. financial issues) and the clients' belief in the usefulness of the treatment [15] [16].

Allen's study on barriers (1994) [17] revealed an interesting dimension of the factors impeding the de-addiction process by organizing them into two categories: the inner and the outer barriers. Inner barriers concern the subjective beliefs of the individual on the existence or non-existence of the addiction problem (problem recognition), the negative emotions mostly experienced through the individual's fear over the opinion of others (stigmatization) and the therapeutic process. The outer barriers include factors such as the accessibility to treatment programs, the features of the health system and a wide range of socio-cultural factors.

The current study is part of a broader systematic research on the psychosocial factors affecting the treatment process within the framework of a residential treatment program. The basic research question revolves around the recovery process and the important data deriving from it.

The first part of the study focuses on presenting the factors facilitating treatment (supports) and the factors undermining it (barriers) as these are experienced by the clients already undergoing treatment. The second part presents an approach of the concept of change during addiction treatment as this is experienced by the clients themselves. The clients' personal understanding of the change concept, i.e. the understanding of the factors or fields which would lead to a positive treatment outcome had the clients been successful in them, is of particular importance to the study.

\section{Method}

\subsection{Participants and Design}

As concerns the sample, the participants were people undergoing treatment within a residential treatment program. This particular program combines the employment of both personal and group therapy through counseling and art expression. The only criterion the participants had to meet was their attendance to the residential treatment program. Besides this criterion, no client was excluded from the study process unless someone did not want to join the study for personal reasons.

Out of all clients, the participants were chosen through a "simple" and random sampling, i.e. every fifth patient on the list was chosen to take part in the program. Altogether 46 clients participated, 37 of them male and 9 female, of age between 19 and 38 years old, with an average age of 30.03 (Std. Deviation: 5.1) years when entering the program. The clients were chosen right from the initial phase of the program, the so called "Consultation Center", during the "Residential Phase" and during the "Social Re-Integration" phase.

The treatment program follows a "three pillars" structure:

1) The outpatient Counseling Centre phase, lasting two to three months, is designed to explore and build on the motivation the client experiences in order to break the substance abuse cycle. It involves participation in a twice weekly psychotherapeutic group that embraces a therapy contract that includes no use of substances, no violence, and no sexual relationships among the clients. The psychotherapeutic group remains a fixture of treatment until the program is completed and is a point of reference for each individual undergoing treatment.

2) The Residential Phase, lasts seven to nine months and comprises the main body of the psychological rehabilitation process. This phase includes individual and group (both supportive and psychodynamic) psychotherapy sessions twice a week, occupational therapy groups, clinical groups (a meeting of all members of the staff 
once a week), sharing in the daily unit care routine (with patients responsible for cleaning, cooking, serving, library work, etc.) and participation in groups operating in accordance with the principles of Narcotics Anonymous.

3) The Social Reintegration phase, which last ten to 12 months and takes place outside the residence. This treatment phase is conducted on a separate premise in downtown Athens and consists of individual and group psychotherapy sessions twice a week (supportive psychotherapy based on the psychodynamic model and adjusted to social re-integration issues such as employment and relationships with parents and society) as well as participation in the daily social re-integration program.

75.3 percent of all participants had been subjected to other addiction treatments in the past. For 67.3 percent of the sample the main abuse substance was heroin, 29.3 percent used various substances simultaneously, while 75.2 percent reported cannabis as the substance setting off drug abuse. The age of initiation of drug abuse was by average 15.5 years and the total duration of use 11 years.

\subsection{Measures}

The research instrument employed in the study was the semi-structured interview, in which the clients were asked to describe the process of change during the treatment and name its most important aspects to them. This particular type of interview was deemed as the most useful for the research measurements, since there were no specific hypotheses to verify but rather issues and questions to answer. Thus, all recorded interviews revolve around the same theme, i.e. the change process during the addiction treatment and the most important parameters relating to it but differing in terms of structure and the questions/answers order. The interviewers were two clinical psychologists, holders of Master of Science Degrees in Clinical Psychology. They were chosen to conduct the interviews due to their previously proven experience in qualitative research.

The interviews were conducted within 28 sessions between the volunteer-researcher and the 46 respondents, coming from 11 different therapy groups. The duration of each interview was circa 40 to 50 minutes. All interviews were taped and transcribed.

\section{Data Analysis}

The analytical method followed was the content analysis (or textual analysis) that includes the coding of the sayings of each client in exclusive and specified categories, which helped summarize and organize the data collected. These categories were formulated by the interview data itself. More specifically, the content analysis was in most cases based on quantifying the replicability of words or phrases of the texts and their analysis on a conceptual level [18]. The coding emerged from the data itself (emergent coding) and there were no predefined categories based on a single theoretical model (a priori coding).

As far as the methodology employed in the following examples is concerned [19], two researchers undertook the task of reading carefully the material collected (data collection) and identifying specified characteristics (codes), out of which they eventually formed a list (concept) addressing the research question. Then, the researchers compared their notes and used the differences spotted in the initial material to manageably code the data (categories). Afterwards, they used their final list of common characteristics to conduct a new coding indicative of the research question, and in the end, they proceeded with the evaluation of the data reliability ( $97 \%$ of accuracy rate, 0.8 on the Cohen's Kappa scale). Following the exact same pattern, the basic coding was complemented by the subcategories [19].

The unit of analysis in this study basically emphasized on the content, meaning and main concept of the narrative. An analytical counting of the words was not deemed as necessary, since the emerging themes were easily recognizable and distinguished. Although conceptual analysis raises several obstacles in extracting themes, the single and specified sample of participants in this case of study (substance abusers undergoing the same treatment program) simplified by far the extraction of meaningful theme categories.

This resulted in having to pay attention both to to the clients' chosen words or phrases and to their specific way of describing the experiences and arguments developed during the interview. Next followed the open coding of the overall text corpus, from which emerged general categories. The categories were then grouped and produced the final categories of findings. During this procedure, as accurately highlighted by Cavanagh (1997) [20], the purpose of coding is not only the selective reduction of the categories but the best possible and accurate description of the phenomenon, in order to better understand it and promote knowledge. In the end, each one of 
the categories were labeled with a code (meaning unit) relating to the same central content of their characteristics.

\section{Results}

Three categories of factors emerged from the interview data analysis: supports, barriers and change assessment.

\subsection{Supports}

The first category includes all factors clients perceive as helpful in treatment, and concern both therapeutic processes/methods requiring the client's participation as well as personal processes mostly occurring during treatment. More specifically, team-work is considered to be the most common supportive factor. Emphasis is being placed on the processes occurring within each therapy group, especially in terms of interaction, contact, support, interest, help, behavioral mirroring, communication, trust, listening, sense of security, co-operation and sharing. Clients tend to refer to these characteristics outside the group too, since they reflect processes observed in people living together within the framework of a residential treatment program and relate to all-group activities. Personal therapy, on the other hand, is the second most referred to supportive factor because it shapes a strong bond between the two sides involves (therapist-patient relationship) and is deemed as the time of deepest and truest expression of emotions. Personal therapists become reference persons for the clients, since they are the only sober persons they can form a relationship with during treatment.

The client's personal will is often being described as a highly important factor contributing to staying in treatment. Determination, willfulness, optimism, high spirits and positive thinking constitute this leading emotion. In this way, the treatment gains a sense of personal choice and responsibility on behalf of the patient, who starts an effort, stays true to it and finally completes it.

Characteristics, such as the general function of the treatment program, thinking processes, brainstorming, reasonable elaboration and analysis and social support, especially on behalf of the addict's family, record lower, yet significant, percentages. Other supports include the trust a patient puts in the concept and processes of treatment and the overall satisfaction gained from the therapy offered. As far as the analysis process is concerned, it is a rather unprecedented experience for the clients because they get in touch with the consequences of their behavior and the ways in which they change. Social support is reckoned, quite expectedly, as particularly helpful to treating addiction. However, the rather low percent of clients claiming to have received enough support from their social surroundings indicates the dubious role this factor can actually play. Table 1 shows the main support factors.

\subsection{Barriers}

The second category the analysis produced includes the barriers clients have to overcome during their substance abuse treatment. This category does not describe the obstacles affecting treatment but rather the steps clients must take while undergoing therapy. These steps are directly connected to the behavioral patterns substance abusers developed — or failed to do so-while they used any kind of substances. The most basic barrier clients refer to is relationships they build either with their fellow clients and staff members of the treatment program or with their relatives and friends. All these relationships are being processed during treatment. According to the clients, this barrier includes the development of trust, living together, denial feelings and parity of communication. Developing honest contacts and communication with others seems to be the very core of the de-addiction process, since clients are asked to do so in a sober state of mind without any "help" or "coverage" from substances. Of course interpersonal relationships are not deemed as a literal barrier but rather as a challenging parameter clients must face from the early stages of treatment.

The second most common barrier is experienced while facing practical issues relating to the therapy process. This specific subcategory concerns mainly the adjustment of the patient to each different phase of the treatment program (Consultation Center, Residential Phase and Social Re-Integration) and the responsibilities that come with it (e.g. intensive therapy within the Residential Phase/adjustment to the community and seeking employment within the Re-Integration phase). Moreover, clients must tackle various legal and financial issues and suspensions from the time when they were using substances, which are often an obstacle to their struggle of beginning a new life. 
Table 1. Supports.

\begin{tabular}{ccc}
\hline Supports & $\mathrm{n}$ & $\%$ \\
\hline Group therapy & 34 & 73.9 \\
Personal therapy & 25 & 54.3 \\
Personal will & 12 & 26.1 \\
General treatment program & 8 & 17.4 \\
Analysis process & 7 & 15.2 \\
Social support & 6 & 13.0 \\
\hline
\end{tabular}

Self-expression and emotional management are further characteristics presenting barriers to clients, especially when suppressed feelings come to surface. In fact, during the first phase of the treatment process the most vivid emotions clients display are pessimism, guilt and stress, which turn into anger, fear, anxiety and a sense of optimism during the second phase. Lastly during the Social Re-Integration phase clients tend to feel nervous but satisfied and optimistic at the same time over the treatment outcome.

Last but not least, addicts consider the process of personal change as challenging; a process, which includes accepting and elaborating on issues that had until now remain locked away in the subconscious, bridging the gap between the past and the present, striving for change and accepting problematic aspects of one's character. Table 2 summarizes the main barriers in treatment.

\subsection{The Concept of Change}

The third and final category the contextual concept analysis produced reflects the way in which clients understand i.e. what does change really mean to them. Changing coincides most definitely effort one puts into better handling difficult situations in life and in managing them more successfully. Additionally, this change is part of a wider change of particular habits and behavioral patterns, which had led or contributed to continuing the substance abuse. Encountering, controlling and with the change of one's way of life and his/her way of thinking as well. This change is being described as the enduring all different kinds of emotions, such as anger, rage, anxiety and fear of the "unknown", is presented as a key component both of the treatment process and the end result of changing. Building relationships, a trust and communication channel with other people is a very significant aspect of the change process. Concluding, some participants attributed a social dimension to the concept of change by mentioning characteristics, such as dignity, the "social face", moral values and social co-existence. These features were not evident during the substance abuse and are very often unprecedented to the clients, since most of them start up using drugs in the developmental period of adolescence. Table 3 shows the main categories of the concept of change.

\section{Conclusions}

The results of this study underline the importance of treating addiction problems within a certain framework organized by community rules and dynamic processes. Clients place emphasis on the concept of communality and are repeatedly referring to relationship issues, thus, pinpointing the crucial role acquaintances, interaction, communication and human touch play in addiction and its cure. Lacking the capacity of building healthy and honest relationships is simultaneously reckoned as one of the basic reasons giving birth to the development of addiction and most probably as one of the conditions that lead to a successful and effective treatment.

Dealing with and managing emotions are described as difficult processes and constitute a means to visualize the change one experiences. Emotions seem to play a constant and highly complicated role in any addiction, since they are both connected to the periods of substance abuse- "euphoria" and "getting high" aim at fighting off negative emotions - and the treatment phase. Zafeiridis (2009) [21] presented in his work the operating mechanism of negative emotions which force the individual to turn to an "ephemeral/illusionary relief" in detail: "When negatively charged emotions are not recognized, not related to their real causes and not understood as responsibilities and parts of one's emotional self, then they become an irrational experience and suggest selfdestructive and unreasonable isolating attitudes, which, of course, need to be justified/rationalized afterwards. This rationalization occurs with the arbitrary ascription of such emotions to a 'hostile' and 'uncontrollable' environment and is the instrument to both justify the present bad emotional shape and reject negative emotions as 
Table 2. Barriers in treatment.

\begin{tabular}{ccc}
\hline Barriers & $\mathrm{n}$ & $\%$ \\
\hline Relationships & 17 & 37.0 \\
Practical issues & 14 & 30.4 \\
Emotional management & 9 & 19.5 \\
Personal change & 8 & 17.4 \\
\hline
\end{tabular}

Table 3. The concept of change.

\begin{tabular}{ccc}
\hline Change & $\mathrm{n}$ & $\%$ \\
\hline Way of life/thinking & 17 & 37.0 \\
Behavior & 10 & 23.9 \\
Emotional encounters & 8 & 17.4 \\
Relationships & 6 & 13.4 \\
Social dimension & 5 & 10.8 \\
\hline
\end{tabular}

irrational by the intellectual self, since they are from now on connected to particular experiences and situations of the present” [21].

Another noteworthy point lies in the recognition of practical issues that seem to bother clients during treatment. These need to be solved in order to ensure the continuance of the effectiveness of the treatment program. Changing one's everyday life and one's behavior is also related to an effective addiction treatment, because this is believed to be necessary for eliciting a positive therapeutic outcome.

Lastly, the energetic individual dimension (personal will, personal change process expressed within the treatment) is of great significance because without it no positive result could be easily achieved. In this way, the client is being somewhat rewarded for his/her personal efforts and willingness to pull through the hard work during treatment.

The current study aims at illustrating some of the most important factors affecting addiction treatment (e.g. emotions) and at confirming the severe impact some already studied factors can have on therapy (e.g. relationships, social support, practical issues). At the same time, the combination of factors emerged relating to the concept of change and demonstrating the complicated nature of addiction and its treatment. The usefulness of the findings for clinical research and work is based upon the special care and attention therapists place on the factors while treating a patient with addiction problems. Realizing and understanding the supports and barriers on behalf of the clients may be the first step towards further processing the factors' qualities. Moreover, the recognition of the factors molding the concept of change could help identify the steps taken in every single stage of treatment towards the desired change. Future studies may reveal more aspects of the changes occurring during treatment or in different kinds of therapy programs and explore all processes relating to each important factor involved.

All in all, it seems that individual and group processes are complementing one another in treatment. The therapeutic process focuses on combining and utilizing the complementary qualities of both processes reflecting real life conditions in a micro level.

The present study had certain limitations to be taken into account, such as the sample selection from a single treatment program. Factors that have been highlighted as important in a residential treatment program (e.g. group therapy) might have not been recognized as such within a different kind of program that does not focus on group processes to the same extent. A larger sample of clients originating from different treatment programs would provide a more accurate and complete image of the important factors affecting the addiction treatment. Furthermore, the content analysis may offer significant information on the object of study but it often does not more than quantify the qualitative data. Perhaps a different qualitative approach (e.g. narrative analysis) would reveal the inner side of the concept of change in addiction treatment.

\section{References}

[1] Winick, C. (1962) Maturing out of Narcotic Addiction. Bulletin on Narcotics, 14, 1-7. 
[2] Waldorf, D. (1983) Natural Recovery from Opiate Addiction: Some Social-Psychological Processes of Untreated Recovery. Journal of Drug Issues, 13, 237-280.

[3] Greaves, G. (1980) An Existential Theory of Drug Dependence. In: Lettieri, D.J., Sayers, M. and Pearson, H.M., Eds., Theories on Drug Abuse, Research Monograph 30, National Institute on Drug Abuse, Rockville, 24-28.

[4] Vaillant, G.E. (1966) A 12-Year Follow-Up of New York Addicts IV: Some Characteristics and Determinants of Abstinence. American Journal of Psychiatry, 123, 573-584.

[5] Vaillant, G.E. (1983) The Natural History of Alcoholism. Harvard University Press, Cambridge.

[6] Wille, R. (1983) Processes of Recovery from Heroin Dependence: Relationship to Treatment, Social Changes and Drug Use. Journal of Drug Issues, 13, 333-342.

[7] Prochaska, J.O., Diclemente, C.C. and Norcross, J.C. (1992) In Search of How People Change: Applications to Addictive Behaviors. American Psychologist, 47, 1102-1114. http://dx.doi.org/10.1037/0003-066X.47.9.1102

[8] Miller, W.R. and Hester, R.K. (1986) Inpatient Alcoholism Treatment: Who Benefits? American Psychologist, 41, 794-805. http://dx.doi.org/10.1037/0003-066X.41.7.794

[9] Costello, R.M. (1975) Alcoholism Treatment and Evaluation II: Collation of Two-Year Follow-Up Studies. International Journal of Addictions, 10, 857-867.

[10] Peele, S. (1985) The Meaning of Addiction: An Unconventional View. Jossey Bass Publishers, San Francisco.

[11] Ravenna, M., Hölzl, E., Kirshler, E., Palmonari, A. and Costarelli, S. (2002) Drug Addicts in Therapy-Changes in Life Space in the Course of One Year. Journal of Community \& Applied Social Psychology, 12, 353-368. http://dx.doi.org/10.1002/casp.688

[12] Felix-Ortiz, M., Salazar, M.R., Gonzalez, J.R., Sorensen, J.L. and Plock, D. (2000) A Qualitative Evaluation of an Assisted Self-Help Group for Drug-Addicted Clients in a Structured Outpatient Treatment Setting. Community Mental Health Journal, 36, 339-350. http://dx.doi.org/10.1023/A:1001983527309

[13] Allen, J. (1984) Correlates of Success in Health Change Efforts. Annual Conference of the American Psychological Association, Toronto, 24-28 August 1984.

[14] Miller, W.R. (2006) Motivational Factors in Addictive Behaviors. In: Miller, W. and Carroll, K., Eds., Rethinking Substance Abuse: What the Science Shows, and What We Should Do about It, Guilford Press, New York, 134-150.

[15] Allen, K. and Dixon, M. (1994) Psychometric Assessment of the Allen Barriers to Treatment Instrument. International Journal of the Addictions, 29, 545-563.

[16] Tucker, J.A., Vuchinich, R.E. and Rippens, P.D. (2004) A Factor Analytic Study of Influences on Patterns of HelpSeeking Treated and Untreated Alcohol Dependent Persons. Journal of Substance Abuse Treatment, 26, 327-242. http://dx.doi.org/10.1016/S0740-5472(03)00209-5

[17] Allen, K. (1994) Development of an Instrument to Identify Barriers to Treatment for Addicted Women, from Their Perspective. International Journal of Addictions, 29, 429-444.

[18] Wilkinson, S. (2003) Focus Groups. In: Smith, J.A., Ed., Qualitative Psychology: A Practical Guide to Research Methods, Sage Publications, London, 184-204.

[19] Smith, J.A., Ed. (2003) Qualitative Psychology: A Practical Guide to Research Methods. Sage Publications, London.

[20] Cavanagh, S. (1997) Content Analysis: Concepts, Methods and Applications. Nurse Researcher, 4, 5-13. http://dx.doi.org/10.7748/nr1997.04.4.3.5.c5869

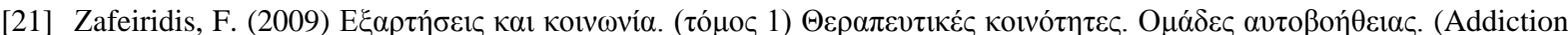
and Society (Vol. 1) Therapy Groups. Self-Help Groups). Kedros Publishing House, Athens, 65-66. 